\title{
Cofactor-mediated conformational control in the bifunctional kinase/RNase Ire1
}

\author{
Alexei V Korennykh ${ }^{1,2,5^{*}}$, Pascal F Egea ${ }^{2,6}$, Andrei A Korostelev ${ }^{3}$, Janet Finer-Moore ${ }^{2}$, Robert M Stroud ${ }^{2}$, \\ Chao Zhang ${ }^{1,4}$, Kevan M Shokat ${ }^{1,4}$ and Peter Walter ${ }^{1,2}$
}

\begin{abstract}
Background: Ire1 is a signal transduction protein in the endoplasmic reticulum (ER) membrane that serves to adjust the protein-folding capacity of the ER according to the needs of the cell. Ire1 signals, in a transcriptional program, the unfolded protein response (UPR) via the coordinated action of its protein kinase and RNase domains. In this study, we investigated how the binding of cofactors to the kinase domain of Ire1 modulates its RNase activity.

Results: Our results suggest that the kinase domain of Ire1 initially binds cofactors without activation of the RNase domain. RNase is activated upon a subsequent conformational rearrangement of Ire1 governed by the chemical properties of bound cofactors. The conformational step can be selectively inhibited by chemical perturbations of cofactors. Substitution of a single oxygen atom in the terminal $\beta$-phosphate group of a potent cofactor ADP by sulfur results in ADPBS, a cofactor that binds to Ire1 as well as to ADP but does not activate RNase. RNase activity can be rescued by thiophilic metal ions such as $\mathrm{Mn}^{2+}$ and $\mathrm{Cd}^{2+}$, revealing a functional metal ion-phosphate interaction which controls the conformation and RNase activity of the Ire1 ADP complex. Mutagenesis of the kinase domain suggests that this rearrangement involves movement of the $\alpha \mathrm{C}$-helix, which is generally conserved among protein kinases. Using X-ray crystallography, we show that oligomerization of Ire1 is sufficient for placing the $\alpha C$-helix in the active, cofactor-bound-like conformation, even in the absence of cofactors.

Conclusions: Our structural and biochemical evidence converges on a model that the cofactor-induced conformational change in Ire1 is coupled to oligomerization of the receptor, which, in turn, activates RNase. The data reveal that cofactor-Ire 1 interactions occur in two independent steps: binding of a cofactor to Ire 1 and subsequent rearrangement of Ire1 resulting in its self-association. The pronounced allosteric effect of cofactors on protein-protein interactions involving Ire1's kinase domain suggests that protein kinases and pseudokinases encoded in metazoan genomes may use ATP pocket-binding ligands similarly to exert signaling roles other than phosphoryl transfer.
\end{abstract}

\section{Background}

The unfolded protein response (UPR) is an intracellular signaling pathway that provides homeostatic feedback regulation between the endoplasmic reticulum (ER) and the gene expression program in the nucleus. To this end, the UPR senses the conditions inside the ER, detecting an imbalance between newly synthesized protein influx and the protein-folding capacity in the ER lumen, and activates a corrective response. When

\footnotetext{
* Correspondence: akorenny@princeton.edu

'Howard Hughes Medical Institute, University of California, San Francisco, 600 16th Street, Room S272, Box 0724, San Francisco, CA 94158, USA Full list of author information is available at the end of the article
}

activated, the UPR drives a broad transcriptional program that adjusts the abundance of the ER [1].

The primary signal transduction device in the UPR is an ER membrane-resident sensor of misfolded proteins, Ire1. Ire1 is conserved from yeast to mammalian cells [2-4]. According to one model, unfolded proteins act as ligands that bind directly to the Ire1 luminal domain $[5,6]$. Another model posits an indirect mode of activation in which the ER-luminal chaperone BiP plays a central role [7]. Both views converge on the concept that oligomerization of the Ire1-luminal domain is crucial for signal propagation across the membrane. Oligomerization of Ire1's luminal domains leads to a local increase

\section{Biomed Central}


in the concentration of Ire1's cytoplasmic kinase and RNase domains, which are tethered to the ER-luminal domain through a transmembrane segment. Recent structural insights suggest that oligomerization activates the cytoplasmic modules by their cooperative assembly into an ordered oligomer [8]. The kinase domains undergo trans-autophosphorylation, which installs phosphate-mediated salt bridges between adjacent Ire1 monomers to stabilize the oligomer. The resulting juxtaposition of Ire1's RNase domains assembles and stabilizes the RNase active site [8,9]. Ire 1 then initiates the unconventional splicing of $H A C 1$ (yeast) or $X B P 1$ (metazoan) mRNA by cleaving either mRNA at two conserved sites to excise an intron $[8,10]$. Transfer RNA ligase completes the mRNA splicing reaction in yeast [4], and an as yet unknown enzyme ligates the exons in metazoans. Intron removal allows the production of the UPR transcription activators Hac1 and XBP1, respectively. The transcription factors upregulate UPR target genes, closing a feedback loop that adjusts the ER's protein-folding capacity according to need.

The kinase domain of Ire 1 is structurally and biochemically similar to that of other protein kinases, including cSrc [11], epidermal growth factor receptor (EGFR) [12], PKR [13], GCN2 [14], cyclin-dependent kinase 2 (CDK2) [9], Aurora [15], cAMP [16] and mitogen-activated protein kinase kinase 1 [17] (Additional file 1, Figures S1 and S2). Notably, binding of nucleotides and synthetic ligands to the kinase domain of Ire1 allosterically activates the Ire1 RNase $[8,18]$. Ire1's RNase activity provides a built-in reporter of kinase conformation, establishing Ire1 as a unique model in which conformational properties of a kinase can be studied separately from the phosphoryl transfer step by using a sensitive and quantitative enzymatic readout.

ADP serves as a cofactor [10] which stimulates RNase activity of Saccharomyces cerevisiae Ire1 in vitro by approximately 200 -fold [8]. It has been proposed that ADP and other ATP pocket ligands stabilize Ire1 in a conformation that forms oligomers [8]. As protein kinases related to Ire1 can assume two globally different conformational states, commonly referred to as "inactive" and "active" [12], Ire1 monomers were thought to exist in an inactive conformation and cofactors were thought to stabilize the active conformation via occupancy of the ATP pocket [18]. This simple model, however, cannot explain the paradoxical observation that ADP has been reported to be a better cofactor than the bulkier ATP [10]. Binding of ATP to the ATP pocket does not fully trigger the RNase-activating conformational change in Ire1, arguing against the model that ATP pocket occupancy is sufficient to lock Ire1 into the active conformation and to activate RNase. In the present study, we carried out a quantitative analysis of Ire1 oligomerization and RNase activation properties with a series of cofactors to resolve this conundrum and to glean insights into the allosteric mechanism by which cofactors control the activity of Ire1 RNase.

\section{Results}

\section{RNase activity quantitatively correlates with Ire1} oligomerization

We observed previously [8] that Ire1 RNase is activated upon high-order oligomerization of Ire1's kinase/RNase domains (Figure 1a). At sufficiently high concentrations, a cytosolic portion of Ire1 (Ire1KR32) forms large-scale oligomers that scatter visible light and display high RNase activity [8]. Ire1KR32 contains Ire1's cytoplasmic kinase and RNase domains, is autophosphorylated as isolated from the Escherichia coli expression host and bears an $\mathrm{N}$-terminal 32-aa extension derived from the linker that connects the Ire1 kinase and transmembrane domains. This $\mathrm{N}$-terminal extension is important for optimal oligomerization and RNase activity of Ire1 [8]. As Ire1KR32 oligomerization resulted in cloudy solutions visible to the naked eye, we explored whether sample transparency could be used to evaluate the oligomer formation quantitatively. We found that turbidity of Ire1KR32 solutions measured as an optical density at $500 \mathrm{~nm}$ (OD500) indeed provides a reliable, quantitative metric of oligomerization (Figure 1b).

As a function of enzyme concentration, the $\mathrm{OD}_{500}$ of Ire1KR32 solutions increased cooperatively by approximately two orders of magnitude in both the absence and presence of the activating cofactor ADP Mg (Figure $1 \mathrm{~b}$, diamonds and circles, respectively). Observed Hill coefficients $(n=3$ for Ire1 ADP Mg and $n=8$ for apoIre1) were in close agreement with those determined in an enzymatic assay that monitors Ire1's RNase activity ( $n=3.5$ for Ire1 KR32 ADP Mg and $n=8$ for apoIre1KR32 [8]) (Figure 1a). In the presence of the ADP $\mathrm{Mg}$ cofactor, both $\mathrm{OD}_{500}$ and RNase profiles shift similarly toward lower Ire1KR32 concentrations, which, together with similar Hill coefficients, indicates that both assays monitor the same physical phenomenon: high-order Ire1 oligomerization.

As shown in an overlay of the $\mathrm{OD}_{500}$ and RNase measurements (Additional file 1, Figure S3), both assays are indeed superimposable. $\mathrm{OD}_{500}$ was below the detection limit at Ire1KR32 concentrations that already displayed activated RNase, indicating that active Ire1KR32 oligomers at these concentrations are too sparse to result in detectable turbidity. By contrast, $\mathrm{OD}_{500}$ values continued to rise after RNase activity reached a kinetic plateau due to the onset of a saturating regime $k_{2}$, as the oligomer concentration exceeded the $K_{\mathrm{m}}$ for RNA binding [19]. The RNase and $\mathrm{OD}_{500}$ readouts therefore complement one another and allow monitoring of the log-linear 


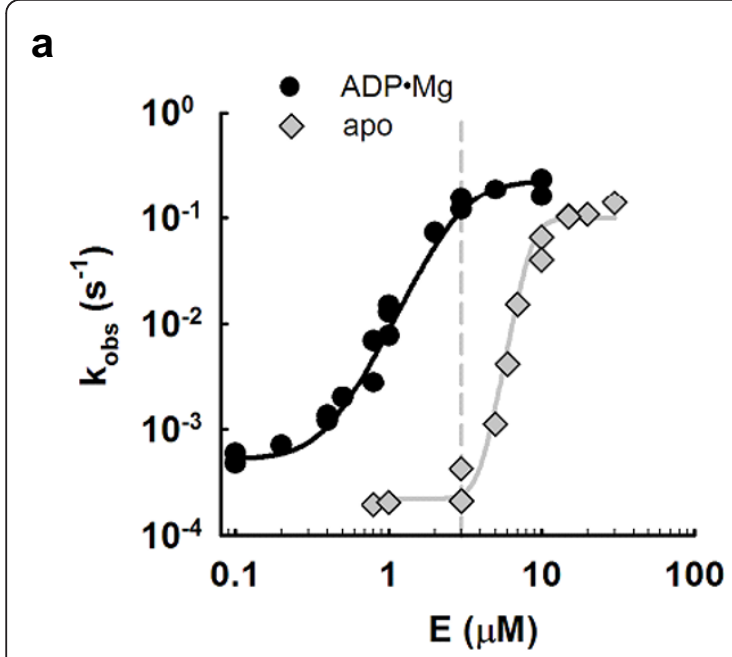

C

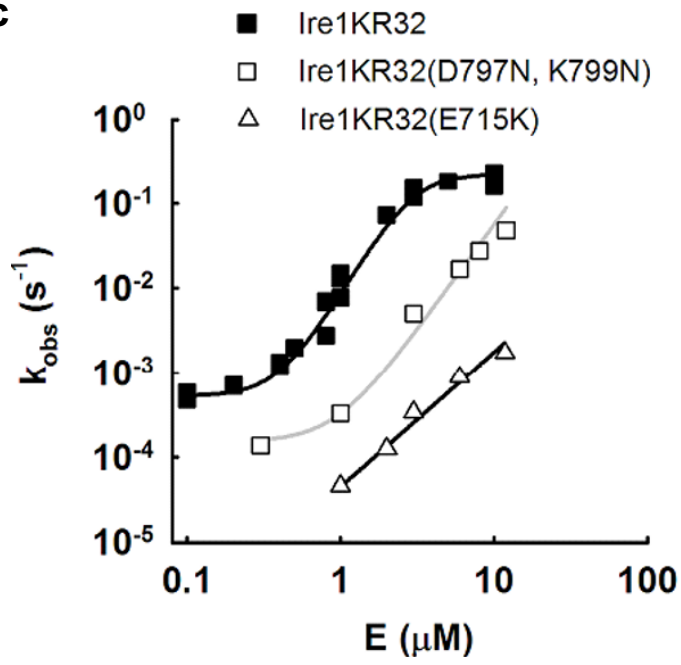

b

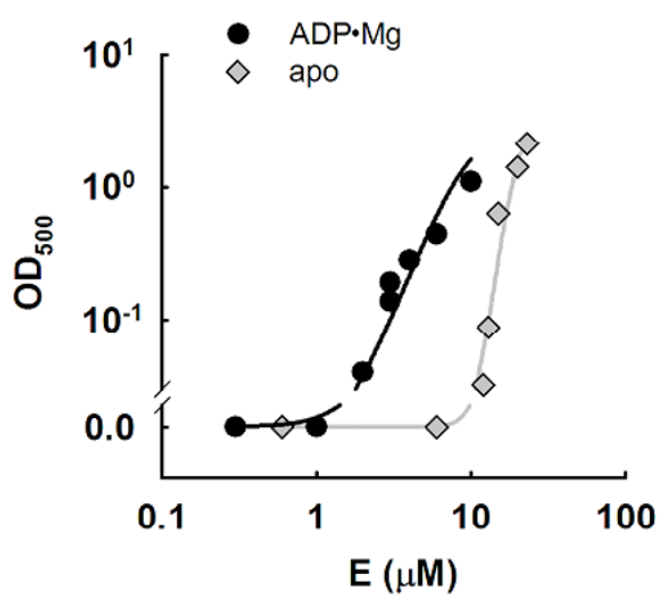

d

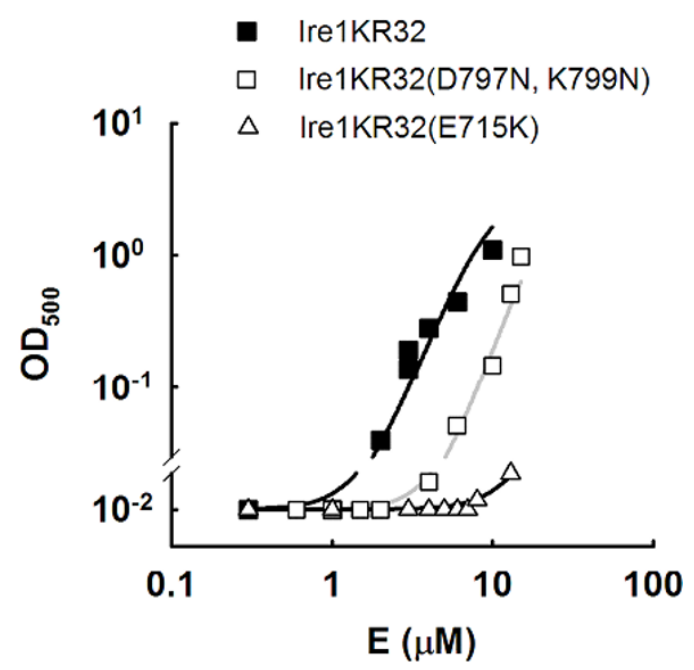

Figure 1 Linking RNase activity and high-order oligomerization of Ire1 kinase/RNase. (a) Cooperative activation of Ire1 RNase with and without 2 mmol ADP Mg [8]. The dashed line marks the standard assay condition at 3 umol Ire1KR32. (b) Opacity at $500 \mathrm{~nm}$ versus concentration of Ire1KR32 with and without 2 mmol ADP Mg. (c) RNase activation profiles for Ire1KR32, Ire1KR32(D797N, K799N) bearing a catalytically disabled, unphosphorylated kinase domain, and for Ire1KR32(E715K). Reactions were conducted as in (a) in the presence of $2 \mathrm{mmol}$ ADP Mg. (d) $\mathrm{OD}_{500}$ response versus concentration of Ire1KR32, Ire1KR32(D797N, K799N) and Ire1KR32(E715K). Assays were conducted as in (b) in the presence of $2 \mathrm{mmol}$ ADP Mg.

oligomerization over a broader range of Ire1 concentrations than either assay in isolation. The RNase activity provides a more sensitive measurement and detects oligomers at concentrations several orders of magnitude smaller than those detectable by $\mathrm{OD}_{500}$ readings.

Recombinantly expressed Ire1KR32 used in these studies contains approximately 20 phosphates [8]. We showed previously that removal of these phosphates by mutagenesis of the kinase active site shifted the activation profile to higher Ire1KR32 concentrations [8]. To further verify that the RNase-based readout of oligomerization and the more direct readout based on $\mathrm{OD}_{500}$ absorbance are in agreement, we measured the concentration dependence of an unphosphorylated variant of Ire1KR32, Ire1KR32(D797N, K799N). This double-mutant carries mutations of residues that are required for $\gamma$-phosphate coordination and catalysis of phosphotransfer but otherwise do not disrupt nucleotide coordination at the ATP pocket. Recombinantly expressed Ire1KR32(D797N, K799N) is entirely unphosphorylated as established by mass-spectrometry, and phosphotransfer-inactive [20]. As shown in Figures 1c 
and $1 \mathrm{~d}$, both RNase activity and $\mathrm{OD}_{500}$ profiles were shifted to higher enzyme concentrations for Ire1KR32 (D797N, K799N). Note that the data include the characterization of an additional Ire1 mutant Ire1KR32 (E715K), which also exhibited a consistent shift in both assays and is discussed below.

Therefore, both RNase and $\mathrm{OD}_{500}$ readouts report robustly on cofactor addition (Figures $1 \mathrm{a}$ and $1 \mathrm{~b}$ ) and perturbations due to mutations in Ire1 (Figures $1 \mathrm{c}$ and 1d). We show below that the equivalency between the optical and enzymatic readouts also extends to all other variables tested, such as chemical substitution in the ADP cofactor and addition of thiophilic metal ions in the presence of an ADP isostere, ADP $\beta$ S. The data in Figure 1 therefore indicate that RNase activity and $\mathrm{OD}_{500}$ provide reliable readouts for Ire1KR32 oligomerization and support the model [8] in which oligomerization and RNase activation are mechanistically coupled.

\section{Activation of Ire1 RNase by different cofactors}

To quantitatively define the potency of different Ire 1 cofactors, we measured the activation of Ire1KR32 RNase in vitro following the site-specific cleavage of a

${ }^{32} \mathrm{P}$-labeled RNA hairpin substrate. We found that at saturating concentrations, nucleotides AMP and ATP both activated Ire1KR32 by approximately tenfold (Figures $2 \mathrm{a}$ and $2 \mathrm{~b}$ ). Notably, although the effects at saturation were similar, the apparent affinity for AMP was tenfold lower than that observed for ATP (Figure 2c). By contrast, ADP bound with an apparent affinity similar to that of ATP, but ADP binding resulted in 20-fold stronger activation of the enzyme (Figures $2 \mathrm{~b}$ and $2 \mathrm{c}$ ). Among other nucleotides tested, only 2'-deoxy(ADP) stimulated Ire1KR32 RNase comparably to ADP (Figure $2 \mathrm{~b})$, although it bound more weakly (Figure $2 \mathrm{c}$ ). The divergence of the apparent cofactor binding constants and maximal stimulation of RNase activity at saturating cofactor concentrations extended to other cofactors (Figures $2 \mathrm{~b}$ and 2c). Thus, surprisingly, cofactor binding and cofactor-induced conformational changes that promote Ire1 oligomerization can be experimentally uncoupled. As binding of the larger ATP molecule fails to activate Ire1KR32 to the same extent as does binding of ADP, resulting in an order-of-magnitude smaller response (Figure 2a), we concluded that mere occupancy of the ATP pocket does not effect optimal Ire1KR32 activation.

To describe different cofactors quantitatively, we defined a "potency of cofactor" $\left(P_{\text {cof }}\right)$

$$
\mathrm{P}_{\text {cof }}=\frac{\mathrm{k}_{\mathrm{obs}}^{\text {Ire } 1 \bullet \text { cofactor }}}{\mathrm{k}_{\mathrm{obs}}^{\text {apo }- \text { Ire1 }}}
$$

as the ratio of the $k_{\text {obs }}$ of Ire 1 with saturating amounts of cofactor bound over the $k_{\text {obs }}$ of apo-Ire1, both measured under standard assay conditions, which we defined as $3 \mu \mathrm{mol}$ Ire1KR32. At this Ire1KR32 concentration, maximum fold stimulation by ADP was achieved (Figure 1a, dashed line; see also [8]). The relationship between the macroscopic conformational parameter $P_{\text {cof }}$ and the microscopic equilibria involved in oligomerization is described in the Discussion section and in greater detail in Additional file 1, Supplementary Analysis 1.

When cofactors are compared using the $P_{\text {cof }}$ metric, the RNase activity of apo-Ire1 defines the baseline with $P_{\text {cof }}=1$. The presence of bound ADP stimulates Ire1KR32 strongly with $P_{\text {cof }}$ approximately 200), whereas the presence of bound AMP or ATP is less effective $\left(P_{\text {cof }}\right.$ approximately 10$)$ (Figure $\left.2 \mathrm{~b}\right)$. Similarly, synthetic small molecules that bind to the nucleotide-binding pocket in Ire1 (Additional file 1, Figure S4) can be described by $P_{\text {cof }}$ (Figures $2 \mathrm{~b}$ and $2 \mathrm{c}$ ). Sunitinib, an ATP-mimicking drug developed as a competitive inhibitor of EGFR kinase, activated Ire1KR32 to a similar extent as AMP and ATP ( $P_{\text {cof }}$ approximately 30 ), whereas APY24 and APY29 activated Ire1 as well as or better than ADP ( $P_{\text {cof }}$ approximately 200 to 300 ) (Figure 2b) [8].

\section{A $\beta$-phosphate-coordinated metal ion facilitates Ire1 oligomerization}

Strikingly, the close ADP-mimic ADP $\beta$ S did not activate Ire1KR32 (Figure $2 \mathrm{~b}$; $P_{\text {cof }}$ approximately 1 ; ADP $\beta S$ contains a sulfur atom that replaces a nonbridging oxygen atom on the $\beta$-phosphate, Figure 3a). This finding was paradoxical because ADP $\beta S$ bound to Ire1 with the same affinity as ADP and ATP, as indicated by its apparent $K_{\mathrm{i}}$ in a competitive inhibition assay of Ire1KR32's ADP-stimulated RNase (Figure 2d) and by measurements of the small RNase stimulation by ADP $\beta S$ in the absence of ADP (Figure 2c). Thus we conclude that the ATP pocket can be occupied by a tightly binding cofactor without activating RNase.

The sulfur substitution in ADP $\beta$ S introduces a "soft" atom, which has a larger covalent radius than that of oxygen. Sulfur does not form strong hydrogen bonds and does not coordinate strongly with "hard" cations such as magnesium $[21,22]$. Elegant "metal specificity switch and rescue" experiments that are well established for ribozymes showed that metal ion coordination disrupted as a result of oxygen-sulfur substitutions can sometimes be restored if a softer metal cation such as $\mathrm{Mn}^{2+}$ is supplied to the reaction [21,22]. Indeed, when we added $\mathrm{Mn}^{2+}$ to the assay buffer, we observed complete restoration of ADP $\beta$ S cofactor activity to the level of ADP Mg (Figure 3b; $P_{\text {cof }}$ approximately 100). 
a

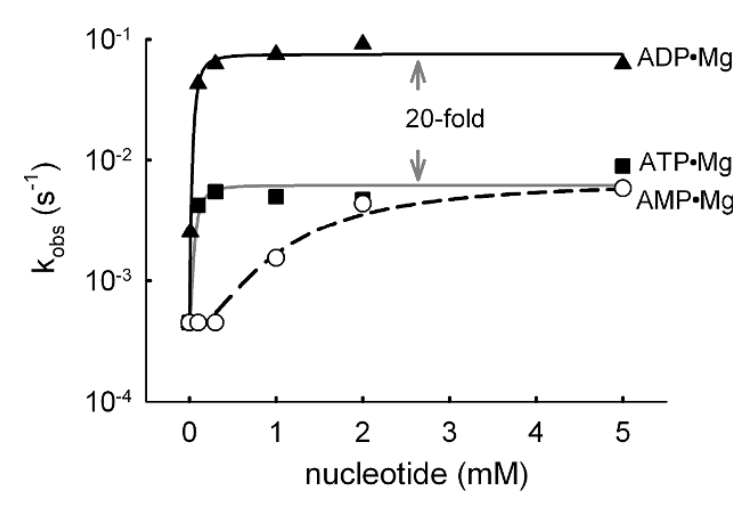

C

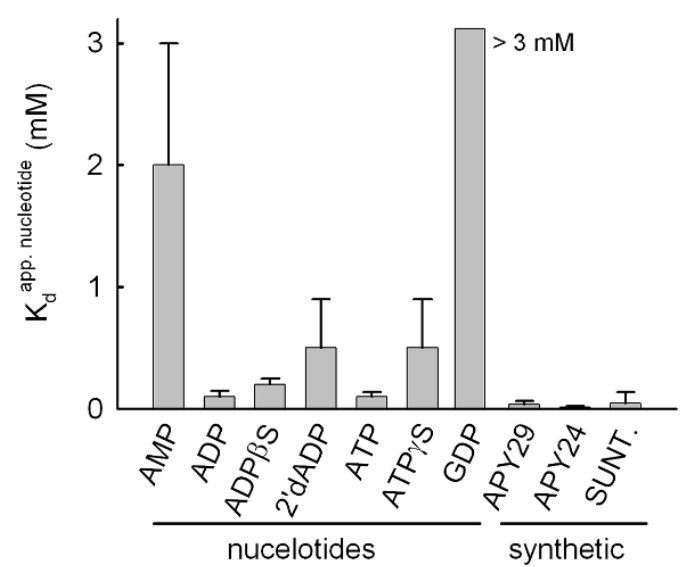

b

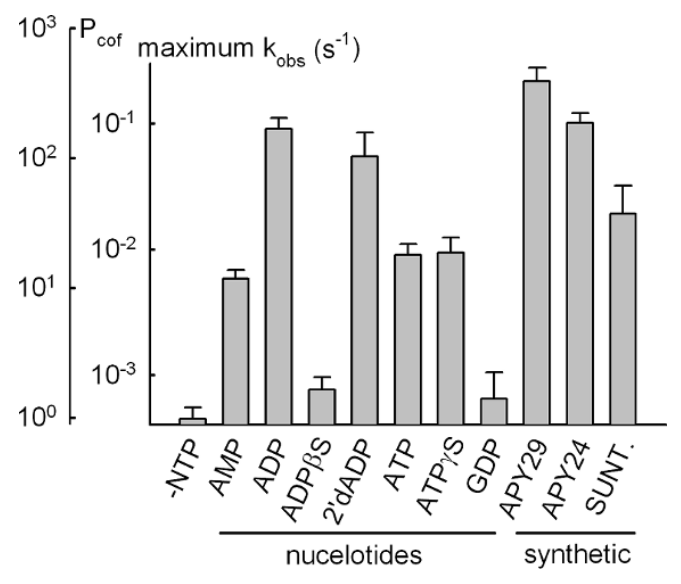

d

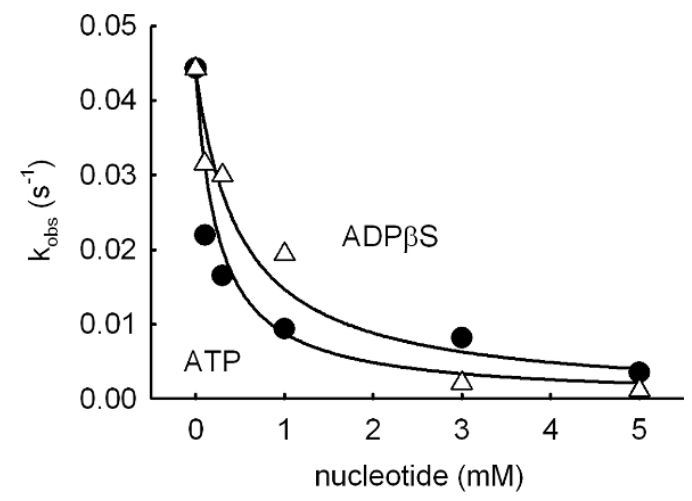

Figure 2 Allosteric activation of Ire1 RNase by ligands binding to the kinase domain. (a) Activation of Ire1KR32 by AMP, ADP and ATP. The $k_{\mathrm{obs}}$ values of RNase activity were determined as a function of nucleotide $\mathrm{Mg}^{2+}$ concentration (see Methods). (b) and (c) Characterization of Ire1 activity with a panel of nucleotides and synthetic ligands. Maximum Ire1 RNase stimulation achieved with cofactors at saturation (b) and apparent cofactor binding constants (c) are shown. The scale of cofactor potency $\left(P_{\text {cof }}\right)$ is indicated in (b). SUNT = sunitinib. Cofactor structures are shown in Additional file 1, Figure S4. (d) Measurement of binding constants for ADP 3 S and ATP via inhibition of a reaction stimulated with $0.2 \mathrm{mmol}$ ADP. $K_{\mathrm{i}}$ values of $0.20 \pm 0.07 \mathrm{mmol}$ and $0.10 \pm 0.04 \mathrm{mmol}$ were determined for ADPBS and ATP, respectively. The data were fit to the binding isotherm and corrected for the $0.2 \mathrm{mmol}$ ADP background. All reactions contained $3 \mu \mathrm{mol}$ Ire $1 \mathrm{KR} 32$ and $2 \mathrm{mmol} \mathrm{Mg}^{2+}$. Error bars show standard errors of binding isotherm fitting.

The rescue was specific for a sulfur-soft metal ion pair because ADP $\beta$ S did not activate Ire1 by more than a few fold in the presence of $\mathrm{Mg}^{2+}$ (Figure $3 \mathrm{~b}$ ) and because $\mathrm{Cd}^{2+}$, another thiophilic metal, also rescued the activity (Additional file 1, Figure S5; $P_{\text {cof }}$ approximately 30). Furthermore, neither $\mathrm{Mn}^{2+}$ nor $\mathrm{Cd}^{2}$ ${ }^{+}$stimulated reactions with oxo-ADP (Figure 3c; Additional file 1, Figure S5). These data strongly point to a functional connectivity between the $\beta$-phosphate of ADP, $\mathrm{Mg}^{2+}$ ion coordination and a conformational change in the Ire1 kinase domain, resulting in activation of Ire1 RNase.
By contrast, such connectivity was not observed for the $\gamma$-phosphate of ATP, which also contacts the $\mathrm{Mg}^{2+}$ ion in crystal structures of protein kinases. ATP $\gamma \mathrm{S}$ bound to Ire1 slightly more weakly than ATP (Figure 2c), but at saturation it functioned indistinguishably from ATP (Figure 2b; $P_{\text {cof }}$ approximately 10 for ATP and ATP $\gamma$ S).

As expected, RNase activation by ADP $\beta S$ and $\mathrm{Mn}^{2+}$ fully coincided with Ire1KR32 oligomerization monitored as $\mathrm{OD}_{500}$. Samples of Ire1KR32 were transparent in the absence of cofactors or in the presence of ADP $\beta S$ or $\mathrm{Mn}^{2+}$ alone. By contrast, white oligomer solutions 
a

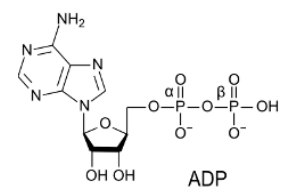

b

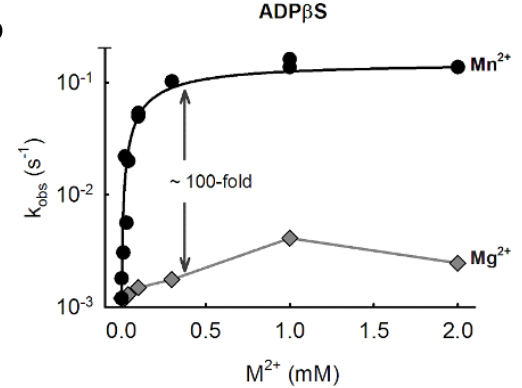

d
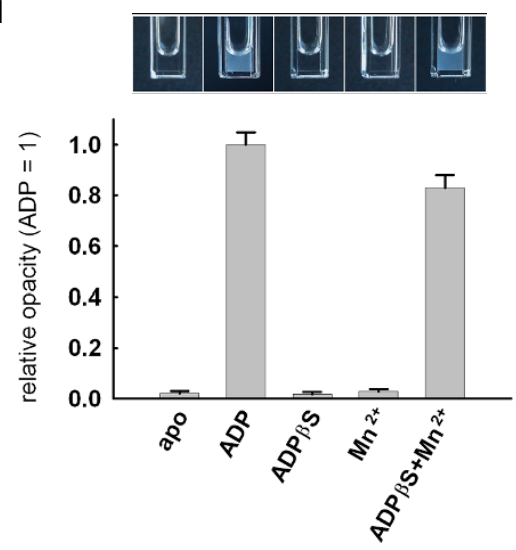

g

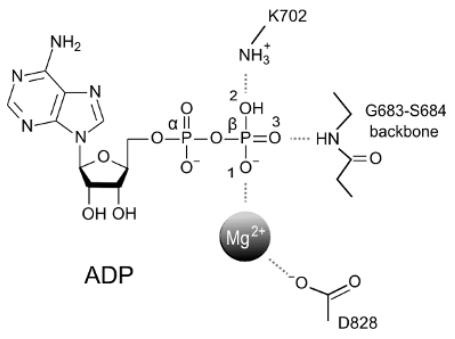

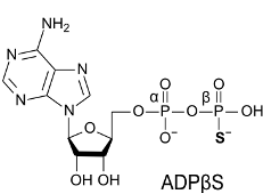

C

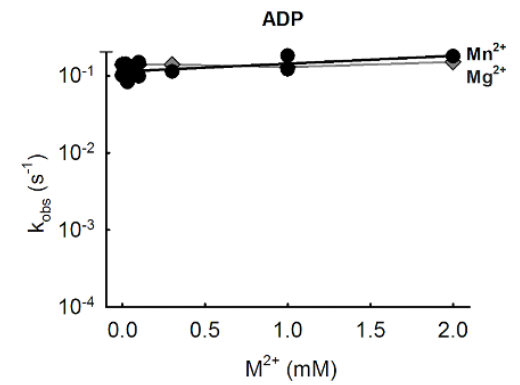

e

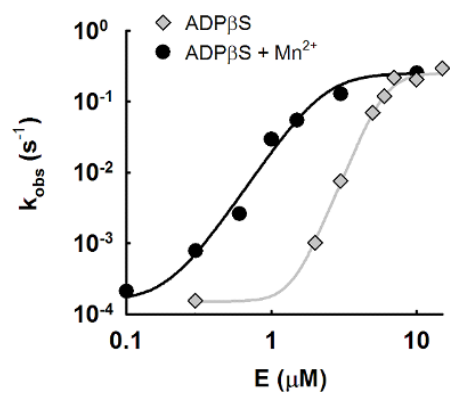

f

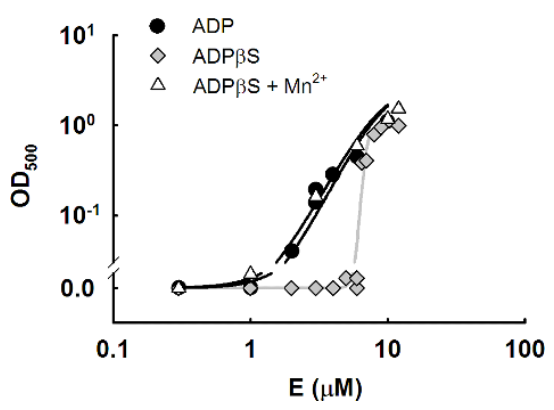

h

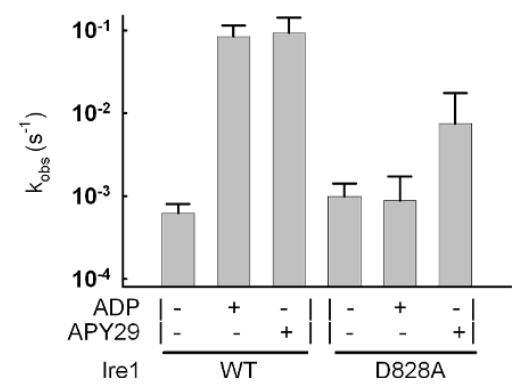

Figure 3 Manganese rescue of Ire1 RNase activity and oligomerization in the presence of ADP $\beta$ S. (a) Structures of ADP and ADP $\beta S$. (b) and (c) The effect of increasing $\mathrm{Mg}^{2+}$ and $\mathrm{Mn}^{2+}$ concentrations on Ire1 RNase activity in the presence of 2 mmol ADPBS (b) or 2 mmol ADP (c). The buffer contained $0.2 \mathrm{mmol} \mathrm{Mg}{ }^{2+}$. All reactions contained $3 \mu \mathrm{mol}$ Ire1KR32. Assays were conducted as described in Figure 1(a)a. (d) Visible oligomerization of Ire1KR32 in the absence of cofactors and in the presence of $1 \mathrm{mmol}$ ADP, $1 \mathrm{mmol}$ ADPBS, $1 \mathrm{mmol} \mathrm{Mn}^{2+}$ and $1 \mathrm{mmol}$ ADPBS

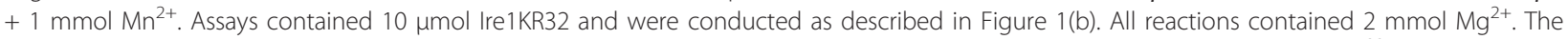
bar chart below the image shows relative opacity of each sample. (e) Cooperative activation of Ire1KR32 RNase assayed using ${ }^{32}$ P-5'-HP21 substrate as described in Figure 1(a). Reactions contained $2 \mathrm{mmol} A D P \beta S, 2 \mathrm{mmol} \mathrm{Mg}{ }^{2+}$ and either 0 or $2 \mathrm{mmol} \mathrm{Mn}^{2+}$ as indicated. (f) $\mathrm{OD}_{500}$ versus concentration of Ire1KR32 in the presence of $2 \mathrm{mmol}$ ADP, $2 \mathrm{mmol}$ ADP $\beta S$ and $2 \mathrm{mmol}$ ADP $\beta S+2 \mathrm{mmol} \mathrm{Mn}^{2+}$. All reactions contained 2 mmol $\mathrm{Mg}^{2+}$. (g) Interactions involving the $\beta$-phosphate in the Ire1 ADP Mg complex (PDB ID 2rio). Note that all three nonbridging oxygen atoms of the $\beta$-phosphate form contacts to either protein residues or $\mathrm{Mg}^{2+}$. (h) The rate constants for Ire1 and Ire1(D828A) in the presence of $\mathrm{ADP} \mathrm{Mg}^{2+}$ and APY29. Error bars show standard errors of single-exponential fitting of corresponding time courses. 
formed in the presence of ADP $+\mathrm{Mg}^{2+}$ or ADP $\beta S+$ $\mathrm{Mn}^{2+}$ (Figure 3d; $2 \mathrm{mmol}$ magnesium background was present in all reactions shown). Measuring RNase activity and $\mathrm{OD}_{500}$ as a function of Ire1KR32 concentration further confirmed that ADP $\beta S$-saturated Ire1KR32 behaved similarly to apo-Ire1KR32. Upon addition of $\mathrm{Mn}^{2+}$, cooperative activation profiles with ADP $\beta S$ became indistinguishable from those obtained with Ire1KR32 and oxo-ADP Mg (Figures 3e and 3f).

Because the $\beta$-phosphate can rotate around the $\alpha-\beta$ bridging bond, replacement of one $\beta$-oxygen atom by sulfur leaves two more oxygen atoms on the $\beta$-phosphate available for coordination of $\mathrm{Mg}^{2+}$. The deleterious effect of the oxygen-to-sulfur substitution in ADP $\beta S$ therefore implies that all three nonbridging oxygen atoms on the $\beta$-phosphate form functionally important interactions, all of which are required for the conformational change in Ire1's kinase domain. Thus, every nonbridging oxygen atom must obligatorily either coordinate $\mathrm{Mg}^{2+}$ or form hydrogen-bonding contacts to the protein. Detrimental effects of the steric bulk introduced by the larger sulfur atom can be disregarded, because ADP $\beta S$ works as well as ADP in the presence of $\mathrm{Mn}^{2+}$.

In the crystal structure of the Ire1 ADP complex (PDB ID 2rio) [9], nonbridging oxygen 1 (Figure 3g) coordinates to $\mathrm{Mg}^{2+}$, nonbridging oxygen 2 forms a hydrogen bond through conserved lysine K702 and nonbridging oxygen 3 forms a hydrogen bond to the backbone of strand $\beta 1$ (GXGXXG motif). This network of connectivity is conserved among protein kinases [23], suggesting that lessons learned from the nucleotide effects on Ire1 may be broadly applicable to other protein kinases.

In Ire1, the $\beta$-phosphate-coordinating $\mathrm{Mg}^{2+}$ ion additionally coordinates to the conserved aspartate D828, which is functionally important for Ire1 RNase activation (Figures $3 \mathrm{~g}$ and $3 \mathrm{~h}$ ). As shown in Figure $3 \mathrm{~h}$, removal of this interaction via a D828A mutation in Ire1KR32 results in a loss of Ire1 responsiveness to ADP $\mathrm{Mg}$, consistent with the importance of the magnesium coordination demonstrated by the studies of ADP $\beta S$ above. Addition of the magnesium-independent cofactor APY29 [8] (Figure 2b and Additional file 1, Figure S4), which replaces the $\beta$-phosphate-magnesium moiety with an aromatic ring, partially activated Ire1KR32(D828A) ( $P_{\text {cof }}$ approximately 10; Figure $\left.3 \mathrm{~h}\right)$. Therefore, the Ire1KR32(D828A) mutant retains the intrinsic ability to become activated by cofactors, and the loss of responsiveness to ADP $\mathrm{Mg}^{2+}$ arises, at least partially, from the impaired magnesium coordination.

On the basis of the data presented so far, we conclude that the $\beta$-phosphate-interactions of ADP with Ire1 kinase form a " $\beta$-phosphate latch". This latch involves a centrally positioned $\mathrm{Mg}^{2+}$ ion that lies at the core of the molecular switch that controls Ire1 oligomerization and thereby its RNase activity. For natural adenosine nucleotides, this latch is engaged via a highly specific hydrogen bonding and metal coordination network. For synthetic ligands, such as APY29, the $\beta$-phosphate latch is bypassed by metal-independent interactions such as hydrophobic and/or hydrogen-bonding contacts.

\section{The position of the $\beta$-phosphate is dynamically linked to the conformation of the kinase domain}

Equivalently placed cofactor-bound $\mathrm{Mg}^{2+}$ ions are found in other protein kinases that are structurally similar to Ire1 [9,11-17], including the closely related and structurally well-characterized cell cycle regulator kinase CDK2. The high structural similarity between CDK2 and Ire1 kinase domains (Additional file 1, Figure S2) suggests that insights gained from CDK2 may help increase the understanding of Ire1 function and vice versa. Comparisons of aligned structures of CDK2 ADP Mg, CDK2 ATP Mg with and without bound cyclin (a CDK2 activator) and apo-CDK2 reveal only a minor displacement (root mean square displacement approximately $1 \AA$ ) of the position of the adenosine base (Figure 4a), yet phosphate atoms of the bound nucleotides move much larger distances. The $\beta$-phosphate in particular stands out as the most rearranged part of the nucleotides, occupying one of two positions that are 4.8 $\AA$ apart. As we discuss below, these two states of the nucleotide and the kinase domain are linked to the rearrangement of the $\alpha \mathrm{C}$-helix common to protein kinases and are indicative of switching CDK2 between its inactive ( $\alpha$ C-helix "out", E51 points outside the ATP pocket) and active ( $\alpha \mathrm{C}$-helix "in", E51 points inside the ATP pocket) conformations (Figure 4b) [12]. The same connection between the $\beta$-phosphate position and the kinase conformation could be established for a wellcharacterized tyrosine kinase cSrc that also has been crystallized in several conformations (Additional file 1, Figure S1a).

Two relevant conclusions arise from published crystal structures of protein kinases. First, ATP can bind to a kinase domain without noticeably altering its inactive conformation, as judged by the unchanged position of the $\alpha \mathrm{C}$-helix in apo-CDK2 and in CDK2 ATP Mg complexes (Figures $4 \mathrm{~b}$ and $4 \mathrm{c}$ ). Therefore, in agreement with our studies on Ire1KR32 and ADP $\beta S$, conformational changes in the kinase domain do not necessarily occur in response to occupancy of the nucleotide binding pocket. Second, transition from the inactive to the active conformation upon the binding of cyclin to CDK2 ATP results in a pronounced (approximately $10 \AA$ ) movement of the $\alpha \mathrm{C}$-helix (Figure $4 \mathrm{~b}, 1$ ), demarcating its switch to the active conformation [12], and in an approximately 
a

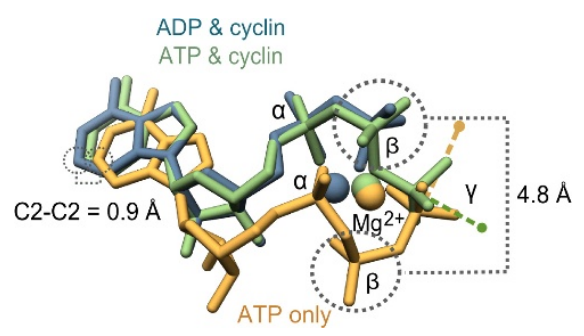

b
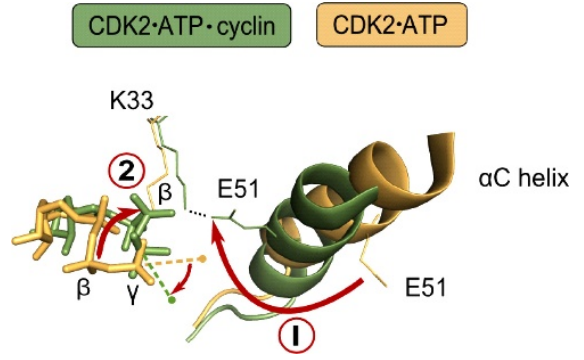

c

$$
\text { CDK2·ADP·cyclin }
$$

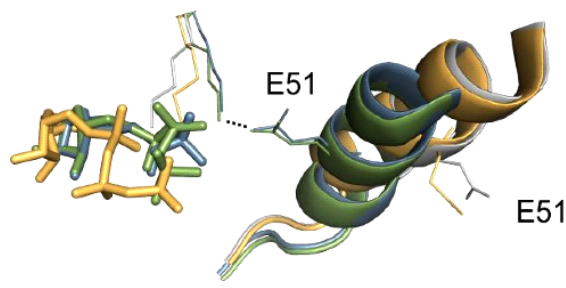

Figure 4 Coupled motion of $\alpha \mathrm{C}$-helix and $\beta$-phosphate in an Ire1-like kinase CDK2. (a) ATP bound to CDK2 (orange trace; CDK2 is in the inactive conformation) is, for most of its atoms, well aligned with ADP and ATP bound to a CDK2 cyclin complex (blue and green traces, respectively; CDK2 is in the active conformation), except for the $\beta$-phosphate, which is displaced by approximately 4.8 $\AA$. (b) Key conformational rearrangements during inactive $\leftrightarrow$ active transition in CDK2. Movement of $\alpha \mathrm{C}$-helix and E51 (1) is coupled to the movement of $\beta$-phosphate (2). The conformational change involves formation of a salt bridge between E51 and K33, formation of an interaction between $\mathrm{K} 33$ and $\beta$-phosphate and rotation of the $\gamma$-phosphate for in-line attack by the $\mathrm{OH}$ group of the substrate (small arrow connecting dashed lines indicates change in geometry) [41]. Coordinates are from PDB ID: 1hcl (apo-CDK2), 1b39 (ATPbound CDK2), 19mz (ATP-bound CDK2 with cyclin) and 1gy3 (ADPbound CDK2 with cyclin). (c) The conformation of the CDK2 kinase domain in the ATP-bound state (inactive conformation with no cyclin bound) is similar to the apo-state (inactive conformation with no cyclin bound) rather than to the ADP-bound state (active conformation with cyclin bound). A corresponding comparison of several additional kinases is shown in Additional file 1, Figure S1.

5 - $\AA$ movement of the $\beta$-phosphate (Figure 4b, 2). This global conformational rearrangement positions a conserved glutamate $\mathrm{E} 51^{\mathrm{CDK} 2}$ to interact through a salt bridge with the conserved lysine $\mathrm{K} 33^{\mathrm{CDK} 2}$, which docks to the $\beta$-phosphate. The docking of the $\beta$-phosphate aligns the $\gamma$-phosphate in the proper geometry for phosphotransfer [24] (Figure 4b, 2).

Our experiments demonstrating the rescue of Ire1KR32 ADP $\beta S$ activity by $\mathrm{Mn}^{2+}$ suggest that $\beta$-phosphate insertion does not occur merely in response to a conformational change in the kinase domain. Instead, it supplies appreciable free energy for stabilizing Ire1 kinase and, by extension, other related protein kinases in the active conformation with the $\alpha C$-helix in the "in" orientation.

The movement involving $\alpha \mathrm{C}$-helix is a dynamic feature of many protein kinases $[12,14,25]$. Important to the work presented here is that the residues for $\beta$ phosphate placement in CDK2 (E51 and K33) are conserved in Ire1 (E715 and K702, respectively). To assess experimentally whether the $\alpha \mathrm{C}$-helix in Ire1 functions in the conformational switch, we probed the predicted salt bridge K702-E715 by mutagenesis. Because K702 is also directly involved in cofactor binding (Figure $4 \mathrm{~b}$ ), we judged that the effects of any K702 mutations could not be interpreted straightforwardly and therefore focused the mutagenesis on E715. We chose to mutate E715 to lysine, creating Ire1KR32(E715K), to minimize perturbations to space filling, local hydrophobicity and H-bonding capability. The E715K mutation introduces a repulsive electrostatic interaction with $\mathrm{K} 702$ and is expected to destabilize the $\alpha \mathrm{C}$-helix "in" conformation.

In agreement with this expectation, the Ire1KR32 (E715K) mutant displayed a vastly decreased RNase activity (Figure 1c, triangles) and did not oligomerize at concentrations at which wild-type Ire1KR32 produced large amounts of oligomers (Figure 1d, triangles versus squares). As the mutational perturbation of $\alpha \mathrm{C}$ helix was expected to affect the phosphotransfer activity of the Ire1 kinase, it was important to rule out that the oligomerization defect of the Ire1KR32(E715K) mutant and its concomitant loss of RNase activity did not result solely from a loss of phosphorylation. The effect of the loss of phosphorylation could be evaluated readily by comparing Ire1KR32(E715K) with Ire1KR32, which would be entirely unphosphorylated. To this end, we used the double-mutant Ire1KR32(D797N, K799N), which has a catalytically inactive kinase domain, purifies with no phosphates attached and retains readily detectable RNase activity [20]. At concentrations that allowed Ire1KR32(D797N, K799N) to oligomerize nearly to the level of wild-type Ire1KR32 and to exhibit nearly maximal RNase activity, Ire1KR32(E715K) showed only trace RNase activity and only trace levels of oligomers in the $\mathrm{OD}_{500}$ readout (Figures $1 \mathrm{c}$ and $1 \mathrm{~d}$ ). These findings confirm that the deleterious effects of the E715K mutation cannot be accounted for even by the complete loss of 
phosphorylation and therefore support the model that E715 controls the conformational properties of Ire1.

This model is also supported by the recent crystal structure of unphosphorylated human Ire1 $\alpha$, which shows human Ire1 $\alpha$ in an inactive conformation. In this structure, the $\beta$-phosphate latch is disengaged, the activation loop and the $\alpha \mathrm{C}$-helix are in the inactive conformation and the salt bridge K599-E612 (corresponding to K702-E715 in yeast Ire1) is disrupted [26]. While formally remaining conjecture, it is very likely that the K599-E612 interaction and the $\beta$-phosphate insertion also occur in the active conformation of human Ire1 $\alpha$, because the active conformations are essentially the same for all protein kinases [27]. According to this view, the functional analyses of Ire1KR32(E715K) presented herein support the conformational similarity of Ire1, CDK2, cSrc, EGFR and related protein kinases inferred from their sequence and structural similarity.

\section{Crystal structure of an Ire1KR32 oligomer with an apo- kinase domain}

The results presented so far suggest a model in which ADP Mg locks Ire1 into the $\alpha \mathrm{C}$-helix "in" conformation, which favors oligomerization and thereby activates RNase. From the principle of coupled equilibria, it follows that oligomerization, if it can be induced without kinase ligand binding, would likewise lock the kinase domain into the active $\alpha \mathrm{C}$-helix "in" conformation. Indeed, high concentrations of apo-Ire1 induce cooperative oligomerization and activation of RNase, illustrating the ability of apo-Ire1 to oligomerize without cofactors (Figures 1a and 1b; see also [8]). Structural insights into this apo-Ire1 oligomer, however, have been missing because to date attempts to crystallize the oligomeric Ire1KR32 in the absence of ATP pocket-binding cofactors have not been successful. In the present study, we circumvented this problem by introducing a new ligand, an oligonucleotide bound to the RNase domain, that resulted in diffracting crystals of IreKR32 in its oligomeric state but with a ligand-free apo kinase domain.

To this end, we used a splice-site mimic oligonucleotide (CCGCAG) containing 2'-deoxy substitutions throughout to prevent its degradation by Ire1. Oligonucleotide-complexed Ire1KR32 crystallized in space group C222 and diffracted to 6.0 to $6.6 \AA$ (Table 1 and Additional file 1, Figure S6). The asymmetric unit contained seven Ire1KR32 molecules. The asymmetric units packed to form the same oligomeric structure as in the 3.2- $\AA$ complex previously crystallized with the smallmolecule activator APY29 in the kinase active site, which crystallized in space group $\mathrm{P} 2{ }_{1} 2_{1} 2$ and had 14 Ire1KR32 molecules per asymmetric unit (PDB ID 3fbv) (Figure 5a). The oligomers in $\mathrm{C} 222$ and $\mathrm{P} 2{ }_{1} 2_{1} 2$ structures pack via a different set of crystallographic contacts (Figure 5a, bottom; note the presence of RNase/RNase interfilament contacts in the $\mathrm{C} 222$ structure but not in the $\mathrm{P} 2{ }_{1} 2{ }_{1} 2$ structure), strongly supporting the notion that the Ire1KR32 oligomer is a stable assembly not resulting from a particular crystal environment. Electron density for the bound substrate was observed in the

Table 1 Data collection and refinement statistics ${ }^{a}$

\begin{tabular}{|c|c|c|c|}
\hline Data collection and refinement & Statistics & Subcategory & Value \\
\hline \multirow[t]{8}{*}{ Data collection } & Cell dimensions & Space group & $\mathrm{C} 222$ \\
\hline & & $a, b, c(\AA)$ & $91.67,580.82,177.99$ \\
\hline & & $\alpha, \beta, \gamma\left({ }^{\circ}\right)$ & $90,90,90$ \\
\hline & & Resolution $(\AA)$ & $97-6.60(6.8-6.60)$ \\
\hline & & $\mid / \sigma(l)$ & $16.62(1.09)$ \\
\hline & & $\mathrm{R}_{\text {pim }}{ }^{\mathrm{b}}(\%)$ & $4.4(88.3)$ \\
\hline & & Number of reflections & $9402(766)$ \\
\hline & & Completeness (\%) & 99.9 (99.9) \\
\hline \multirow[t]{9}{*}{ Data refinement } & Number of atoms (in one monomer) & Protein & 3399 \\
\hline & & Resolution $(\AA)$ & $97-6.60$ \\
\hline & Rigid body (with NCS) & Angles $\left({ }^{\circ}\right)$ & 0.925 \\
\hline & & Bonds $(\AA)$ & 0.006 \\
\hline & & $R_{\text {work }} / R_{\text {free }}$ & $0.2863 / 0.3170$ \\
\hline & Simulated annealing (with NCS) & $\mathrm{t}\left({ }^{\circ} \mathrm{K}\right)$ & 1,000 \\
\hline & & Angles $\left(^{\circ}\right)$ & 1.197 \\
\hline & & Bonds $(\AA ̊)$ & 0.007 \\
\hline & & $\mathrm{R}_{\text {work }} / \mathrm{R}_{\text {free }}$ & $0.2731 / 0.3451$ \\
\hline
\end{tabular}

${ }^{\mathrm{a}}$ Refinement was done using rigid body protocol. Simulated annealing was not used in final analysis, except for calculations of omit maps. Values for the highest resolution shell are shown in parentheses. ${ }^{b}$ Precision-indicating merging R-factor [42].

Definitions: NCS - non-crystallographic symmetry; $R_{\text {pim }}$ - precision-indicating merging factor; $R_{\text {work }}$ - crystallographic residual factor; $R_{\text {free }}$ - free crystallographic residual factor. 
a

\section{Ire1•APY29 complex}

space group $\mathrm{P} 2{ }_{1} 2{ }_{1} 2$

14 molecules (asymmetric unit)

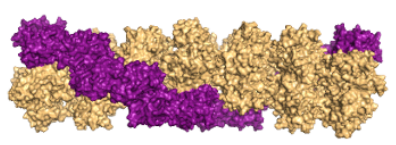

filament packing in crystal

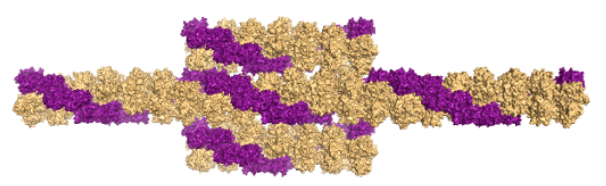

Ire1•DNA complex

(apo-kinase)

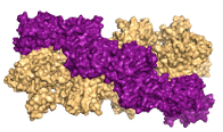

space group C222

7 molecules

(asymmetric unit) filament packing in crystal

b

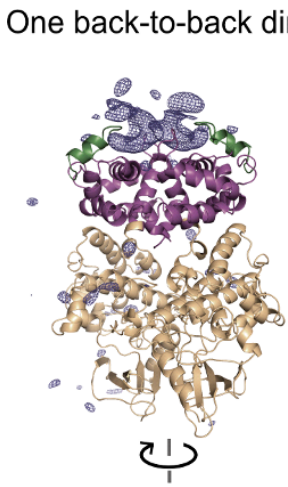

2-fold axis

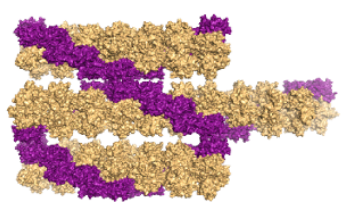

c

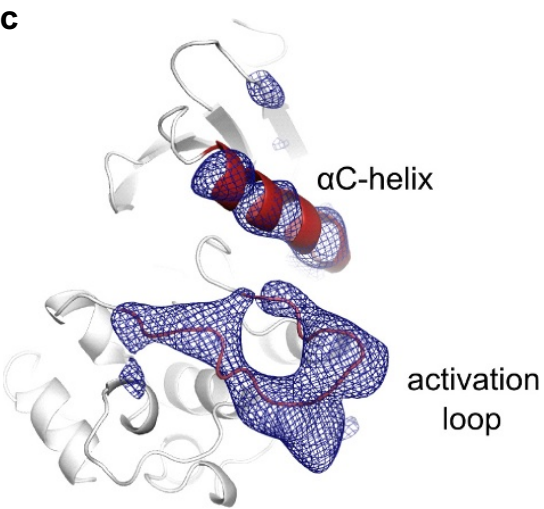

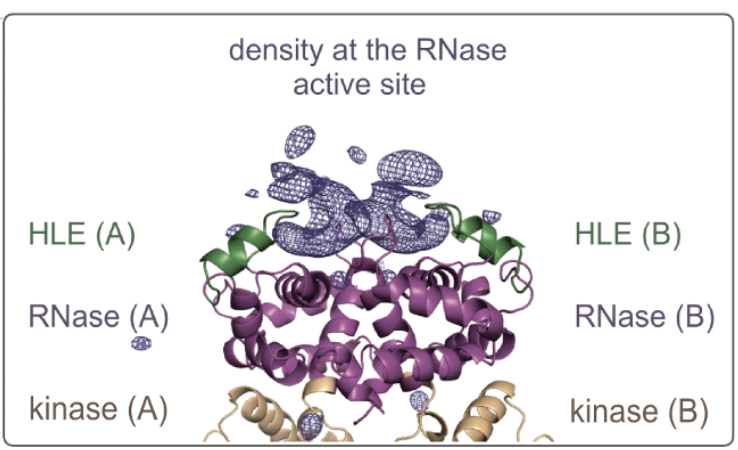

kinase $(A)$

d

aC helix

CDK2·ADP. cyclin

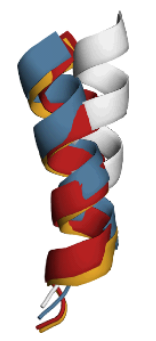

apo-CDK2

Figure 5 Crystal structure of the Ire1KR32 oligomer obtained without ligands in the ATP pocket. (a) Ire1 kinase/RNase forms an oligomer with the synthetic ligand APY29 (crystal P2,2,2) and with a 6-mer DNA oligonucleotide that served as a noncleavable mimic of Ire1's RNA substrate (crystal C222). The sequence dCdCdGdCdAdG of the oligonucleotide is derived from the splice site of HAC1 mRNA. The filaments in the $\mathrm{P}_{2} 2_{1} 2$ and in the C222 structures pack using entirely different sets of crystal contacts. (b) Simulated annealing (2,000 K) NCS-averaged $F_{\text {obs }}$ $F_{\text {calc }}$ difference map of the twofold symmetric density in the RNase active site (contoured at $5.5 \sigma$ ). (c) Simulated annealing (2,000 K) NCSaveraged $F_{\text {obs }}-F_{\text {calc }}$ omit map for the $\alpha C$-helix (contoured at $5.5 \sigma$ ) and for the activation loop (contoured at $4.5 \sigma$ ) in the C222 structure (red). Omit maps were calculated with $\alpha$ C-helix and activation loop residues deleted from all seven monomers prior to refinement. Analogous maps calculated by simulated annealing without NCS restraints are shown in Additional file 1, Figure S7. (d) Superposition of CDK2 and Ire1KR32 in the apo-bound state (this work) and the ADP-bound state (PDB ID 2rio) shows that the $\alpha$ C-helix of apo-lre1 KR32 (in the C222 structure) occupies the same conformation as in Ire1 ADP and in CDK2 ADP cyclin complexes, which is distinct from that in the inactive apo-CDK2 structure. 
RNase active site (Figure 5b). Its implications for the catalysis of RNA cleavage are discussed in a separate publication [19].

Although at 6.6- $\AA$ resolution it is not possible to identify positions of individual side chains, secondary structural elements can be resolved, as evidenced by lowresolution crystal structures of other macromolecules [28], especially if a high-resolution structure can be used for phasing. Sevenfold noncrystallographic symmetry averaging greatly enhanced the effective quality of the Ire1-oligonucleotide complex density map (Figures $5 \mathrm{c}$ versus Additional file 1, Figure S7). A cross-validated SigmaA test [29] showed that useful and statistically significant diffraction spans to approximately $6.0 \AA$. The simulated-annealing omit electron density maps, in which the model bias for the $\alpha \mathrm{C}$-helix and the activation loop regions is minimized, demonstrate unambiguously that the $\alpha \mathrm{C}$-helix and the activation loop, the two most dynamic parts of kinases, are in the same position as they were in two previous structures, in which either ADP Mg or the synthetic activator APY29 was bound (PDB ID 2rio[9] and PDB ID 3fbv[8]) (Figures 5c and $5 \mathrm{~d}$ and Additional file 1, Figure S7). These observations are in agreement with the model that oligomerization of apo-Ire1KR32 requires the $\alpha \mathrm{C}$-helix to be in the "in" position. On the basis of these findings, it is likely that oligomerization plays the same role in controlling Ire1 conformation as cyclin binding does in CDK2 and dimerization does in EGFR [12], such that binding of a protein partner stabilizes the $\alpha \mathrm{C}$-helix in the "in" position, even when the kinase active site is empty.

\section{Discussion}

Protein kinases are dynamic enzymes that couple catalysis to pronounced conformational changes. Studies of their dynamics are limited by the intrinsic difficulty of uncoupling enzymatic phosphoryl transfer from conformational properties. In the present study, we exploited the unique facets of Ire1 biology to overcome this shortcoming. The approach was made possible by Ire1's unique property to form easily quantified high-order homo-oligomers upon activation and by Ire1's RNase domain, which provides a natural built-in reporter of the kinase domain conformation. Conformational changes in Ire1's kinase domain modulate the enzyme's predisposition to form oligomers, in which Ire1-Ire1 contacts across multiple interfaces activate Ire1's RNase activity.

The propensity of Ire1 for activation is modulated by three parameters: (1) its local concentration, (2) its phosphorylation state and (3) its interactions with cofactors in the ATP binding site of Ire1's kinase module. In its physiological setting, the local concentration of the cytoplasmic Ire1 kinase/RNase module is responsive to the protein-folding conditions inside the ER lumen. As unfolded proteins accumulate there, the Ire1's luminal domain oligomerizes, thereby concentrating the covalently tethered kinase/RNase domains on the other side of the membrane. This concentration event is thought to provide the primary switch leading to Ire1 activation. In this view, trans-autophosphorylation would ensue following juxtaposition of the kinase domains. The phosphorylation state of Ire 1 is a dynamic parameter that changes over time and is thought to contribute to a molecular timer. Initial phosphorylation events on the activation loop lead to phosphorylated side chains that form stabilizing salt bridges to neighboring Ire1 molecules in the active oligomer [8], whereas subsequent hyperphosphorylation events appear to promote Ire1 turnoff, perhaps because of oligomer-destabilizing charge repulsion effects [20].

Cofactor binding to the ATP pocket of the kinase domain is expected to provide another regulatory input that strongly modulates the sensitivity of the switch to integrate UPR signaling with the physiological state in the cell's cytoplasm. In particular, it is intriguing to speculate that UPR may be modulated by the ADP/ (ATP+AMP) ratio due to the 20-fold higher $P_{\text {cof }}$ of ADP compared to ATP and AMP. Up to an order of magnitude change in the ADP/ATP ratio has been reported during glucose starvation in pancreatic $\beta$ cells and during apoptosis [30,31]. If this were also the case during the UPR, an increase in the relative ADP level would sensitize Ire1, requiring lower concentrations of unfolded proteins to activate the UPR. An alternative possibility could be that an unknown stress-induced small-molecule metabolite with high $P_{\text {cof }}$ and low $K_{\text {cof }}$, other than nucleotides, could serve biological roles in modulating Ire1 activity via binding in the ATP pocket. Work in mammalian systems suggests that Ire 1 can be activated independently of an accumulation of unfolded proteins in the ER lumen, perhaps by utilizing such a mechanism to shift the oligomerization activation threshold [32]. It has been shown that, in addition to the ATP pocket, Ire1 has a different pocket in the RNase domain and that binding of quercetin to this pocket stimulates Ire1 RNase [33]. This finding opens up a possibility that, in addition to the kinase-binding ligands, ligands binding to the RNase domain of Ire1 contribute to modulation of Ire1 signaling.

Phosphorylation of Ire1 considerably affects its responsiveness to cofactors. In the presence of ADP, fully phosphorylated Ire1KR32 exhibits a 40-fold stronger $P_{\text {cof }}$ (approximately 200) compared to Ire1KR32 (D797N, K799N) lacking phosphates ( $P_{\text {cof }}$ approximately 5) (data not shown). Therefore, phosphorylation not only promotes Ire1 oligomerization but also "primes" the receptor for sensing ATP pocket-binding cofactors. 
Notably, unlike $\mathrm{P}_{\text {cof }}$, binding of cofactors to Ire1 does not appear to depend on Ire1 phosphorylation, as both Ire1KR32 and Ire1KR32(D797N, K799N) exhibit similar $K_{\text {cof }}$ values with ADP (data not shown). The effect of phosphorylation on Ire1 sensitivity to cofactors has also been observed during the study of the quercetin pocket [33]. These observations suggest that modulation of Ire1 signaling by cofactors may be physiologically most important after the UPR has been initiated, amplifying the activity of Ire1 molecules that have already been phosphorylated.

By measuring the Ire1 RNase activity in response to kinase-bound small molecules, we resolved two independent steps in cofactor-Ire1 interactions: cofactor binding and the conformational response to a bound cofactor. Noting the wide spectrum of potencies of different cofactors at saturation, $\mathrm{P}_{\text {cof }}$, we propose a simple model in which cofactors bound in the ATP pocket shift an equilibrium between an inactive and an active conformation of Ire1 (Figure 6). In the model, the inactive conformation "O" corresponds to that of a free Ire1 monomer that has the $\alpha \mathrm{C}$-helix in the inactive, "out" position also observed for other protein kinases. The active conformation "I" corresponds to that of Ire1 oligomers with the $\alpha \mathrm{C}$-helix in the active, "in" position. The conformational equilibrium between the "O" and "I" states depends strongly on the chemical nature of the bound cofactor. Whereas some cofactors, such as ADP $\beta$ S, bind without noticeably perturbing this equilibrium, other natural and synthetic cofactors effect an equilibrium shift of over two orders of magnitude. The effect of the cofactors on the oligomerization equilibria (Figures 6a versus 6b) is summarized in the free energy diagram shown in Figure 6c. According to our model shown in Figure 6c, cofactors lower the free energy of the "monomer I" state (compare the "monomer I" states on the solid and the dashed lines), ultimately resulting in an equilibrium shift toward oligomerization (compare the "oligomer I" states on the solid and the dashed lines).

Quantitative manifestations of the two steps, apparent cofactor affinity $K_{\text {cof }}$ and cofactor potency $P_{\text {cof }}$, do not correlate (Figure $2 \mathrm{~b}$ vs. Figure $2 \mathrm{c}$ ), suggesting that they arise from distinct (perhaps partially overlapping) subsets of molecular interactions. The ADP/ADP $\beta$ S comparison (Figure 2) indicates that the kinase hingecontacting face [8] of the cofactors serves for binding, whereas the K702/E715-contacting face (Figure 4b and Additional file 1 Figure S4) modulates the kinase conformation and could be exploited for designing potent synthetic Ire1 activators. Our findings show that Ire1, as already known for other protein kinases, can exist with the ATP pocket filled by cofactors while remaining in the inactive conformation. With certain cofactors such as ADP $\beta$, this functional state could dominate in solution, a notion previously unappreciated in studies of Ire1.

The macroscopically measured parameters $K_{\text {cof }}$ and $P_{\text {cof }}$ are composite derivatives of the microscopic constants $K_{\mathrm{d}}^{1}, K_{\mathrm{d}}^{2}$ and $K_{\mathrm{d}}^{\text {oligomer }}$. (Figure 6 and Additional file 1, Supplementary Analysis 1). The $K_{\text {cof }}$ value depends largely on (and is equal to, when $K_{\mathrm{d}}^{2}$ is large) the true microscopic binding constant $K_{\mathrm{d}}^{1}$. The $P_{\text {cof }}$ value depends only on $K_{\mathrm{d}}^{2}$ and $K_{\mathrm{d}}^{\text {oligomer }}$ and not on the binding constant $K_{\mathrm{d}}^{1}$. Therefore, $P_{\text {cof }}$ is indeed a quantitative metric of conformational responsiveness to bound cofactor, uncoupled from the cofactor binding step, and describes the apparent pressure pushing the kinase domain toward the active conformation with the $\alpha \mathrm{C}$ helix "in" position. For ADP, $P_{\text {cof }}$ arises predominantly because of the $\beta$-phosphate-magnesium insertion into the kinase domain ( $\beta$-phosphate latch), which is functionally defined herein using the metal specificity switch approach.

Synthetic molecules lacking a $\beta$-phosphate equivalent altogether can achieve the same goal in the absence of $\mathrm{Mg}^{2+}$ ions by inserting bulky moieties in the $\beta$-phosphate position. In agreement with this model, sunitinib (Additional file 1, Figure S4), which is predicted to occupy the $\beta$-phosphate position only partially [8], has a relatively low $P_{\text {cof }}$ (approximately 30 ) (Figure $2 \mathrm{~b}$ ).

In CDK2, productive docking of the ATP $\beta$-phosphate in the latched position is facilitated by an external effector, cyclin, that stabilizes the $\alpha \mathrm{C}$-helix "in" conformation extrinsically [12]. A conceptually similar mechanism is employed by EGFR, which dimerizes using one EGFR monomer to stabilize another EGFR monomer in the $\alpha C$-helix "in" conformation [12]. Ire1 does not require a cyclin equivalent, but instead uses homo-oligomerization to stabilize the $\alpha \mathrm{C}$-helix "in" conformation.

\section{Conclusions}

We have characterized an allosteric mechanism by which cofactor binding to the ATP pocket of the Ire1 kinase domain activates the receptor's RNase domain. Our studies of Ire1KR32(E715K) demonstrate that the K702-E715 salt bridge is important for $\alpha \mathrm{C}$-helix positioning and Ire1 activation. In combination, the structural rearrangements in CDK2 (Figure 4) and the presented functional and structural observations with Ire1 converge on a model in which predisposition toward the active conformation of the kinase domain (defined by the position of the $\alpha \mathrm{C}$-helix) is tightly coupled to $\beta$-phosphate docking of bound nucleotide (a $\beta$-phosphate latch). Suboptimal $\beta$-phosphate docking, as in Ire1 ADP $\beta$ S, Ire1 ATP $\mathrm{Mg}$ or CDK2 ATP Mg, does 
a
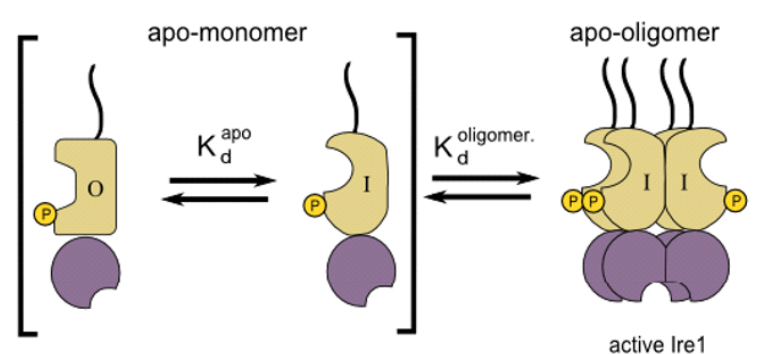

b

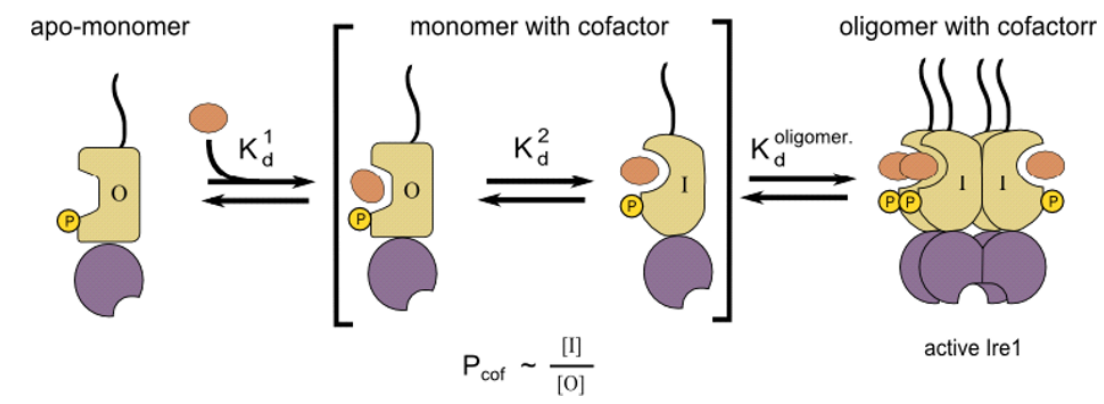

C

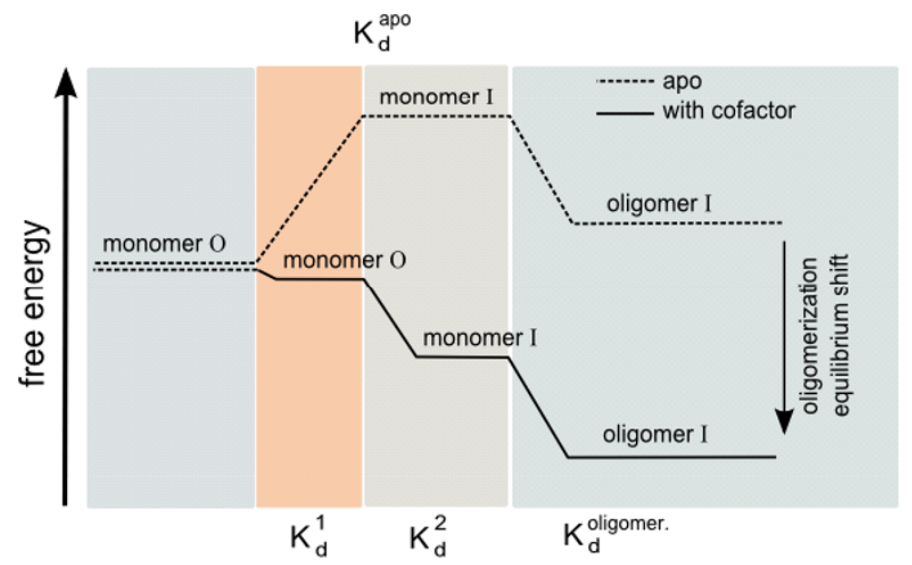

Figure 6 Model of cofactor action during Ire1 oligomerization and activation. (a) Two-step model for oligomerization and activation of apo-Ire1. (b) Three-step model for cofactor-stimulated Ire1 activation involving equilibrium of two distinct cofactor-bound Ire 1 species. The macroscopic parameter $P_{\text {cof }}$ depends on the ratio of cofactor-bound Ire1 populations in "inactive" conformation (marked as " $\mathrm{O}$ ", which designates the $\alpha$ C-helix in the "out" position seen in the inactive conformation of CDK2) and in "active" conformation required for the oligomer assembly (marked as "I", which designates the $\alpha$ C-helix in the "in" position seen in the active conformation of CDK2). The macroscopic apparent cofactor binding constant $K_{\text {cof }}$ depends on the elementary constants $K_{\mathrm{d}}^{1}$ and $K_{\mathrm{d}}^{2}$, whereas the cofactor potency $P_{\text {cof }}$ depends on the $K_{\mathrm{d}}^{2}$ and $K_{\mathrm{d}}^{\text {oligomer }}$ (Additional file 1, Supplementary Analysis 1). (c) Schematic free-energy diagram for the cofactor-independent and the cofactor-facilitated Ire1 oligomerization that corresponds to the schemes shown in (a) and (b). The shift in the oligomerization equilibrium arises from the stabilization of monomer "l" by cofactors.

not preclude the nucleotide from binding, but results in the predominantly inactive conformation and, for Ire1, a correspondingly low $P_{\text {cof }}$ value.

For CDK2, Ire1, cSrc, EGFR and perhaps numerous other protein kinases that use $\alpha C$-helix movement for conformational control, interactions with bound ligands in the ATP pocket may be strongly allosterically coupled to the inactive $\leftrightarrow$ active transition and may influence the surface where these kinases bind external effectors. For Ire1, cofactors produce an orders of magnitude shift in the oligomer population, demonstrating that ligands can drive protein-protein interactions involving the kinase/RNase module of Ire1. Analogously, cofactormediated conformational tuning of protein kinases related to Ire1 might be deployed to recruit their binding partners and allow protein kinases and catalytically 
inactive pseudokinases encoded in metazoan genomes to function as signaling conformational switches independent from ATP hydrolysis. The paradoxical in-transactivation of RAF kinase and extracellular signal-regulated kinase by kinase inhibitors [34,35] apparently arises from such a mechanism.

\section{Methods}

\section{Experimental errors}

Observed rate constants were always determined from reaction time courses. Kinetic parameters were reproduced two or more times and had day-to-day variability typically within twofold. This uncertainty is small compared to all effects presented in this work as significant. Errors and experimental uncertainties are indicated where applicable.

\section{Ire1 expression and purification}

Ire1KR32 and its mutants were expressed as glutathione $S$-transferase (GST) fusion proteins using pGEX-6P-2 plasmid (GE Healthcare, Waukesha, WI) and codoncompensated E. coli (BL21-CodonPlus(DE3)-RIPL Competent Cells; Stratagene, Santa Clara, CA) as described previously [8]. In brief, expression was performed at room temperature for four hours after isopropyl- $\beta$-Dthiogalactopyranoside induction. Cells were lysed using the EmulsiFlex-C3 homogenizer (Avestin Inc. Ottawa, Ontario, Canada) and proteins were purified by performing an affinity column chromatography with subsequent cleavage of the GST tag by using PreScission Protease (GE Healthcare, Waukesha, WI). All protein mutants were fractionated by gel filtration on an S200 column (GE Healthcare, Waukesha, WI) to approximately $99 \%$ purity. Proteins stocks (10 to $20 \mathrm{mg} / \mathrm{mL}$ ) were stored at $-80^{\circ} \mathrm{C}$ in the presence of $5 \%$ glycerol.

\section{RNA substrates}

RNA oligonucleotides were purchased from Dharmacon Inc. (Lafayette, $\mathrm{CO}$ ), labeled at the 5'-terminus using $\mathrm{T} 4$ polynucleotide kinase and ${ }^{32}$ P-ATP (PerkinElmer-NEN, Waltham MA) and purified by $20 \%$ PAGE that allowed single-nucleotide resolution as described previously [8].

\section{Ire1 RNase cleavage assay}

The kinetics of RNA cleavage were assessed as described previously [8]. Typically, reactions were carried out in a $10-\mu \mathrm{L}$ volume at $30^{\circ} \mathrm{C}$. Reactions were started by adding $1 \mu \mathrm{L}$ of ${ }^{32} \mathrm{P}$-labeled RNA to $9 \mu \mathrm{L}$ of premixture containing $20 \mathrm{mmol}$ 4-(2-hydroxyethyl)-1-piperazineethanesulfonic acid (HEPES), pH 7.4, $70 \mathrm{mmol} \mathrm{NaCl}, 2 \mathrm{mmol}$ $\mathrm{MgCl}_{2}$ (unless indicated otherwise), $4 \mathrm{mmol}$ dithiothreitol (DTT), 5\% glycerol and cofactors as indicated. The reactions contained $\leq 1 \mathrm{pmol}$ radioactively ${ }^{32} \mathrm{P}$-labeled RNA and were conducted under single-turnover conditions. Unless noted otherwise, the Ire1 concentration was $3 \mu \mathrm{mol}$. The enzyme concentration was determined from the Ire1KR32 sequence by using absorbance at $280 \mathrm{~nm}\left(\mathrm{e}_{280}=40.8 \times 10^{3} \mathrm{~mol}^{-1} \mathrm{~cm}^{-1}\right)$ calculated using the Electronic Lab Notebook program http://biochemlabsolutions.com/ELN/ELN.html. Reactions were quenched at time intervals with $6 \mu \mathrm{L}$ of stop solution containing $10 \mathrm{~mol}$ urea, $0.1 \%$ SDS, $0.1 \mathrm{mmol}$ ethylenediaminetetraacetic acid, $0.05 \%$ xylene cyanol and 0.05\% bromophenol blue. Samples were analyzed by performing $10 \%$ to $20 \%$ PAGE, and gels were scanned using the Typhoon 9400 scanner (Molecular Dynamics-GE Healthcare, Waukesha, WI) and quantified using ImageQuant image analysis software (Molecular Dynamics-GE Healthcare, Waukesha, WI and GelQuant.NET software http://biochemlabsolutions.com/GelQuantNET.html. The data were plotted and fit in SigmaPlot software (Systat Software Inc., Chicago, IL) to exponential curves to determine observed rate constants and to hyperbolic curves to determine binding constants.

\section{Optical assay of Ire1 oligomerization}

Ire1KR32 oligomerization was assayed in a reaction buffer containing $20 \mathrm{mmol}$ HEPES, pH 7.4, $70 \mathrm{mmol} \mathrm{NaCl}$, $2 \mathrm{mmol} \mathrm{MgCl}_{2}, 4 \mathrm{mmol}$ DTT and 5\% glycerol. Cofactors and $\mathrm{Mn}\left(\mathrm{CH}_{3} \mathrm{COO}\right)_{2}$ were added as indicated for each experiment. The transparency of the samples containing higher concentrations of Ire1 visibly changed immediately upon adding the enzyme. Samples were allowed to sit for 15 minutes to allow for complete Ire1 oligomerization. The OD of the samples was then measured at room temperature $\left(22^{\circ} \mathrm{C}\right)$ at $500 \mathrm{~nm}$ on a UVvisible spectrophotometer (Amersham Ultrospec 3300 Pro; Amersham-GE Healthcare, Waukesha, WI). OD $_{500}$ was obtained after subtraction of baseline absorbance from the buffer free of Ire1. Sample absorbance did not change upon repeated scans of the same sample, indicating that the oligomerization had reached equilibrium under the conditions of the experiments.

The oligomer of Ire1KR32 has a uniform absorbance over 300 to $800 \mathrm{~nm}$, exhibiting higher absorbance at shorter wavelengths. We selected $\lambda=500 \mathrm{~nm}$ for practical considerations, because at this wavelength proteins do not absorb light and the relevant range of Ire1KR32 concentrations $(0.1$ to $10 \mu \mathrm{mol})$ produces a signal within the optimal instrument range ( 0.01 to $1 \mathrm{OD})$. The same titration profiles were obtained not only by reading OD but also by measuring light scattering at a $90^{\circ}$ angle at excitation and emission wavelengths of $500 \mathrm{~nm}$ on a FluoroLog-3 fluorometer (HORIBA Jobin Yvon, Edison, NJ).

\section{Ire1KR32 (dCdCdGdCdAdG) complex crystallization}

The Ire1KR32 (dCdCdGdCdAdG complex was prepared by mixing Ire1KR32 and dCdCdGdCdAdG (Integrated 
DNA Technologies, Coralville, IA). When performed at a relatively low $\mathrm{NaCl}$ concentration $(300 \mathrm{mmol}$ or less), addition of the oligonucleotide caused profound precipitation of the oligomeric complex, as observed upon the addition of ADP Mg [8]. The oligonucleotide thus apparently promoted the formation of Ire1KR32 oligomers analogous to ADP Mg and to APY29. The precipitate readily redissolved upon a slight increase in $\mathrm{NaCl}$ concentration, indicating that the oligomerization reaction was salt-dependent and readily reversible. Crystallization was conducted in hanging drops using a stock solution of a premade complex containing Ire1KR32 (12 $\mathrm{mg} / \mathrm{mL}$ ) and $0.6 \mathrm{mmol} \mathrm{2'-deoxy-CCGCAG.} \mathrm{The} \mathrm{well}$ solution contained 0.12 mol sodium citrate (pH 6.5), 7\% PEG 3350 and $4 \%$ glucose. Single crystals grew overnight and were cryoprotected in a well solution containing $25 \%$ ethylene glycol. The crystals belong to orthorhombic space group C222, distinct from the orthorhombic space group $\mathrm{P} 2{ }_{1} 2_{1} 2$ previously reported for Ire1KR32 oligomer with bound APY29. Crystallization of a variety of other nucleotides and protein constructs was also tested but produced either inferior crystals or no crystals.

\section{$\mathrm{X}$-ray data collection and analysis}

Diffraction from Ire1 (2'-deoxy-CCGCAG) crystals was collected on Beamline 8.3.1 (Advanced Light Source, Lawrence Berkeley National Laboratory, Berkeley, CA, USA; http://www-als.lbl.gov/index.php/beamlines/ beamlines-directory/118-831.html at an X-ray wavelength of $1.115872 \AA$ and an oscillation angle of $1^{\circ}$. The data were indexed, integrated and scaled using the XDS X-Ray Detector software package (MPI for Medical Research, Heidelberg, Germany; http://xds. mpimf-heidelberg.mpg.de/) [36] (Table 1). Five percent of the reflections were marked as a test set $\left(R_{\text {free }}\right)$. A molecular replacement solution was found using Phaser Crystallographic Software [37] starting from monomer $\mathrm{C}$ of PDB ID $3 \mathrm{fbv}$ as a search model. Seven copies of monomer $C$ were found in the asymmetric unit, and the resulting 7-mer of Ire1KR32 was used for rigid body refinement in PHENIX software [38]. Simulated annealing was attempted and, as expected for 6.6- $\AA$ resolution, resulted in an excessive separation between residual factor $R_{\text {work }}$ and free residual factor $R_{\text {free }}$ (Table 1 ). Simulated annealing was therefore used only for calculation of unbiased omit maps $(2,000 \mathrm{~K})$. Fourier $\sigma_{\mathrm{A}}$-weighted [39] $F_{\text {obs }}-F_{\text {calc }}$ difference maps were used for interpretation of the parts of the model missing from the starting structure. Electron density and structure were analyzed and graphed in Coot (Crystallographic Object-Oriented Toolkit) software [40] and PyMOL software http:// www.pymol.org/. The sevenfold non-crystallographic symmetry (NCS) of the model was used to improve the local quality of the electron density maps by symmetry averaging. However, all elements of Ire1 secondary structure were clearly visible without NCS. Coordinates have been deposited into the Protein Data Bank (PDB:3SDM; http://www.rcsb.org/pdb/ search/structidSearch.do?structureId=3SDM.

\section{Additional material}

Additional file 1: Supplementary figures, tables and analyses.

\section{Acknowledgements}

We thank the staff of Beamline 8.3.1 and Chris Waddling of the University of California, San Francisco molecular structure group for help in data collection and structure analysis. We are grateful to David Morgan and Natalia Jura for their critical reading of the manuscript and valuable suggestions. AVK was supported by a Jane Coffin Childs Fund fellowship and the Howard Hughes Medical Institute. RMS, JFM and PFE were supported by National Institutes of Health grant R01 GM60641. PW and KMS are investigators at the Howard Hughes Medical Institute.

\section{Author details}

${ }^{1}$ Howard Hughes Medical Institute, University of California, San Francisco, 600 16th Street, Room S272, Box 0724, San Francisco, CA 94158, USA. ${ }^{2}$ Department of Biochemistry and Biophysics, University of California, San Francisco, Genentech Hall, 600 16th Street, San Francisco, CA 94158, USA. ${ }^{3}$ Department of Biochemistry and Molecular Pharmacology and RNA

Therapeutics Institute, University of Massachusetts Medical School, 364 Plantation Street, Worcester, MA 01605, USA. ${ }^{4}$ Department of Cellular and Molecular Pharmacology, University of California, San Francisco, Genentech Hall, 600 16th Street, San Francisco, CA 94158, USA. ${ }^{5}$ Department of Molecular Biology, Princeton University, 216 Schultz Laboratory, Princeton, NJ 08544, USA. ${ }^{6}$ Department of Biological Chemistry, University of California, Los Angeles, 310 BSRB, Los Angeles, CA 90095, USA.

\section{Authors' contributions}

AVK designed and conducted the biochemical experiments and prepared protein and RNA constructs. AVK and PFE carried out Ire1 crystallization and diffraction data collection. JFM conducted initial diffraction data analyses. AVK and AAK carried out model building and refinement. CZ prepared synthetic Ire1 modulators. PW, KMS and RMS supervised the work. AVK and PW wrote the manuscript. All authors read and approved the final manuscript.

\section{Received: 6 May 2011 Accepted: 6 July 2011 Published: 6 July 2011}

\section{References}

1. Ron D, Walter P: Signal integration in the endoplasmic reticulum unfolded protein response. Nat Rev Mol Cell Biol 2007, 8:519-529.

2. Cox JS, Walter P: A novel mechanism for regulating activity of a transcription factor that controls the unfolded protein response. Cell 1996, 87:391-404.

3. Shamu CE, Walter P: Oligomerization and phosphorylation of the Ire1p kinase during intracellular signaling from the endoplasmic reticulum to the nucleus. EMBO J 1996, 15:3028-3039.

4. Sidrauski $C$, Cox JS, Walter P: tRNA ligase is required for regulated mRNA splicing in the unfolded protein response. Cell 1996, 87:405-413.

5. Credle JJ, Finer-Moore JS, Papa FR, Stroud RM, Walter P: On the mechanism of sensing unfolded protein in the endoplasmic reticulum. Proc Natl Acad Sci USA 2005, 102:18773-18784.

6. Pincus D, Chevalier MW, Aragón T, van Anken E, Vidal SE, El-Samad H, Walter P: BiP binding to the ER-stress sensor Ire1 tunes the homeostatic behavior of the unfolded protein response. PLoS Biol 2010, 8:e1000415.

7. Zhou J, Liu CY, Back SH, Clark RL, Peisach D, Xu Z, Kaufman RJ: The crystal structure of human IRE1 luminal domain reveals a conserved 
dimerization interface required for activation of the unfolded protein response. Proc Natl Acad Sci USA 2006, 103:14343-14348.

8. Korennykh AV, Egea PF, Korostelev AA, Finer-Moore J, Zhang C, Shokat KM, Stroud RM, Walter P: The unfolded protein response signals through high-order assembly of Ire1. Nature 2009, 457:687-693.

9. Lee KP, Dey M, Neculai D, Cao C, Dever TE, Sicheri F: Structure of the dual enzyme Ire1 reveals the basis for catalysis and regulation in nonconventional RNA splicing. Cell 2008, 132:89-100.

10. Sidrauski $C$, Walter $P$ : The transmembrane kinase Ire1p is a site-specific endonuclease that initiates mRNA splicing in the unfolded protein response. Cell 1997, 90:1031-1039.

11. Getlik M, Grütter C, Simard JR, Klüter S, Rabiller M, Rode HB, Robubi A, Rauh D: Hybrid compound design to overcome the gatekeeper T338M mutation in cSrc. J Med Chem 2009, 52:3915-3926.

12. Zhang X, Gureasko J, Shen K, Cole PA, Kuriyan J: An allosteric mechanism for activation of the kinase domain of epidermal growth factor receptor. Cell 2006, 125:1137-1149.

13. Dar AC, Dever TE, Sicheri F: Higher-order substrate recognition of elF2a by the RNA-dependent protein kinase PKR. Cell 2005, 122:887-900.

14. Dey M, Cao C, Sicheri F, Dever TE: Conserved intermolecular salt bridge required for activation of protein kinases PKR, GCN2, and PERK. J Biol Chem 2007, 282:6653-6660.

15. Bayliss R, Sardon T, Vernos I, Conti E: Structural basis of Aurora-A activation by TPX2 at the mitotic spindle. Mol Cell 2003, 12:851-862.

16. Yang J, Ten Eyck LF, Xuong NH, Taylor SS: Crystal structure of a CAMPdependent protein kinase mutant at 1.26A: new insights into the catalytic mechanism. J Mol Biol 2004, 336:473-487.

17. Warmus JS, Flamme C, Zhang LY, Barrett S, Bridges A, Chen H, Gowan R, Kaufman M, Sebolt-Leopold J, Leopold W, Merriman R, Ohren J, Pavlovsky A, Przybranowski S, Tecle H, Valik H, Whitehead C, Zhang E: 2Alkylamino- and alkoxy-substituted 2-amino-1,3,4-oxadiazoles-O-Alkyl benzohydroxamate esters replacements retain the desired inhibition and selectivity against MEK (MAP ERK kinase). Bioorg Med Chem Lett 2008, 18:6171-6174.

18. Papa FR, Zhang C, Shokat K, Walter P: Bypassing a kinase activity with an ATP-competitive drug. Science 2003, 302:1533-1537.

19. Korennykh A, Korostelev A, Pascal E, Finer-Moore J, Zhang C, Shokat K, Stroud R, Walter P: Structural and functional basis for RNA cleavage by Ire1. BMC Biol

20. Rubio C, Pincus D, Korennykh A, Schuck S, El-Samad H, Walter P: Homeostatic adaptation to endoplasmic reticulum stress depends on Ire1 kinase activity. J Cell Biol 2011, 193:171-184.

21. Forconi M, Lee J, Lee JK, Piccirilli JA, Herschlag D: Functional identification of ligands for a catalytic metal ion in group I introns. Biochemistry 2008, 47:6883-6894

22. Hougland JL, Kravchuk AV, Herschlag D, Piccirilli JA: Functional identification of catalytic metal ion binding sites within RNA. PLOS Biol 2005, 3:e277.

23. Hanks SK, Hunter T: Protein kinases 6 . The eukaryotic protein kinase superfamily: kinase (catalytic) domain structure and classification. FASEB J 1995, 9:576-596.

24. Brown NR, Noble ME, Endicott JA, Johnson LN: The structural basis for specificity of substrate and recruitment peptides for cyclin-dependent kinases. Nat Cell Biol 1999, 1:438-443.

25. Pawson $\mathrm{T}$, Kofler M: Kinome signaling through regulated protein-protein interactions in normal and cancer cells. Curr Opin Cell Biol 2009, 21:147-153.

26. Ali MM, Bagratuni T, Davenport EL, Nowak PR, Silva-Santisteban MC, Hardcastle A, McAndrews C, Rowlands MG, Morgan GJ, Aherne W, Aherne W, Collins I, Davies FE, Pearl LH: Structure of the Ire1 autophosphorylation complex and implications for the unfolded protein response. EMBO J 2011, 30:894-905.

27. Jura N, Zhang X, Endres NF, Seeliger MA, Schindler T, Kuriyan J: Catalytic control in the EGF receptor and its connection to general kinase regulatory mechanisms. Mol Cell 2011, 42:9-22.

28. Brunger AT, DeLaBarre B, Davies JM, Weis WI: X-ray structure determination at low resolution. Acta Crystallogr D Biol Crystallogr 2009, 65:128-133.

29. Ling $H$, Boodhoo A, Hazes B, Cummings MD, Armstrong GD, Brunton JL, Read RJ: Structure of the shiga-like toxin I B-pentamer complexed with an analogue of its receptor Gb3. Biochemistry 1998, 37:1777-1788.
30. Salt IP, Johnson G, Ashcroft SJ, Hardie DG: AMP-activated protein kinase is activated by low glucose in cell lines derived from pancreatic $\beta$ cells, and may regulate insulin release. Biochem J 1998, 335:533-539.

31. Bradbury DA, Simmons TD, Slater KJ, Crouch SP: Measurement of the ADP: ATP ratio in human leukaemic cell lines can be used as an indicator of cell viability, necrosis and apoptosis. J Immunol Methods 2000, 240:79-92.

32. Martinon F, Chen X, Lee AH, Glimcher LH: TLR activation of the transcription factor XBP1 regulates innate immune responses in macrophages. Nat Immunol 2010, 11:411-418.

33. Wiseman RL, Zhang Y, Lee KP, Harding HP, Haynes CM, Price J, Sicheri F, Ron D: Flavonol activation defines an unanticipated ligand-binding site in the kinase-RNase domain of IRE1. Mol Cell 2010, 38:291-304.

34. Poulikakos Pl, Zhang C, Bollag G, Shokat KM, Rosen N: RAF inhibitors transactivate RAF dimers and ERK signalling in cells with wild-type BRAF. Nature 2010, 464:427-430.

35. Hatzivassiliou G, Song K, Yen I, Brandhuber BJ, Anderson DJ, Alvarado R, Ludlam MJ, Stokoe D, Gloor SL, Vigers G, Morales T, Aliagas I, Liu B, Sideris $S$, Hoeflich KP, Jaiswal BS, Seshagiri S, Koeppen H, Belvin M, Friedman LS, Malek S: RAF inhibitors prime wild-type RAF to activate the MAPK pathway and enhance growth. Nature 2010, 464:431-435.

36. Kabsch W: Automatic processing of rotation diffraction data from crystals of initially unknown symmetry and cell constants. J App/ Cryst 1993, 26:795-800.

37. McCoy AJ, Grosse-Kunstleve RW, Adams PD, Winn MD, Storoni LC, Read RJ: Phaser crystallographic software. J Appl Cryst 2007, 40:658-674.

38. Adams PD, Grosse-Kunstleve RW, Hung LW, loerger TR, McCoy AJ, Moriarty NW, Read RJ, Sacchettini JC, Sauter NK, Terwilliger TC: PHENIX: building new software for automated crystallographic structure determination. Acta Crystallogr D Biol Crystallogr 2002, 58:1948-1954.

39. Read RJ: Coefficients for maps using phases from partial structures with errors. Acta Cryst 1986, A42:140-149.

40. Emsley P, Cowtan K: Coot: model-building tools for molecular graphics. Acta Crystallogr D Biol Crystallogr 2004, 60:2126-2132.

41. Welburn JP, Tucker JA, Johnson T, Lindert L, Morgan M, Willis A, Noble ME, Endicott JA: How tyrosine 15 phosphorylation inhibits the activity of cyclin-dependent kinase 2-cyclin A. J Biol Chem 2007, 282:3173-3181.

42. Weiss MS: Global indicators of X-ray data quality. J Appl Cryst 2001, 34:130-135.

doi:10.1186/1741-7007-9-48

Cite this article as: Korennykh et al:: Cofactor-mediated conformational control in the bifunctional kinase/RNase Ire1. BMC Biology 2011 9:48.

\section{Submit your next manuscript to BioMed Central and take full advantage of:}

- Convenient online submission

- Thorough peer review

- No space constraints or color figure charges

- Immediate publication on acceptance

- Inclusion in PubMed, CAS, Scopus and Google Scholar

- Research which is freely available for redistribution

Submit your manuscript at www.biomedcentral com/submit
C Biomed Central 